\title{
In-vivo Targeting of Liver Lesions with a Navigation System based on Fiducial Needles
}

\author{
L. Maier-Hein ${ }^{1}$, A. Tekbas ${ }^{2}$, A. Seitel ${ }^{1}$, F. Pianka ${ }^{2}$, S. A. Müller ${ }^{2}$, S. Schawo ${ }^{3}$, \\ B. Radeleff ${ }^{3}$, R. Tetzlaff ${ }^{1,4}$, A. Franz ${ }^{1}$, A.-M. Rau ${ }^{1,4}$, I. Wolf ${ }^{1}$, H.-U. Kauczor ${ }^{4}$, \\ B. M. Schmied ${ }^{2}$, H.-P. Meinzer ${ }^{1}$ \\ ${ }^{1}$ German Cancer Research Center, Div. of Medical and Biological Informatics \\ ${ }^{2}$ University of Heidelberg, Dept. of General, Abdominal and Transplant Surgery \\ ${ }^{3}$ University of Heidelberg, Dept. of Diagnostic Radiology \\ ${ }^{4}$ German Cancer Research Center, Dept. of Radiology \\ 1.maier-hein@dkfz-heidelberg.de
}

\begin{abstract}
We assessed the targeting precision of a previously reported needle-based soft tissue navigation system in-vivo. Eight $2 \mathrm{ml}$ agar nodules were implanted into the livers of two swine as tumor models. One medical expert with experience in punctures and one non-expert used the navigation system to target each nodule twice. The targeting error, which includes the system error and the user error, was defined as the distance between the applicator tip and the center of gravity of the lesions in computed tomography (CT) control scans and was $5.3 \pm 2.2 \mathrm{~mm}(\mathrm{n}=16)$ for the expert, $3.2 \pm 1.3 \mathrm{~mm}(\mathrm{n}=16)$ for the non-expert, and $4.2 \pm 2.1 \mathrm{~mm}(\mathrm{n}=32)$ averaged over both subjects. A robust targeting precision of the order of magnitude of $4 \mathrm{~mm}$ could improve the treatment standard for CT-guided minimally invasive interventions in the liver dramatically.
\end{abstract}

\section{Introduction}

Computed tomography $(\mathrm{CT})$ guided minimally invasive procedures in the liver such as tumor biopsy and thermal ablation therapy frequently require the targeting of hepatic structures that are subject to breathing motion. Unfortunately, commercially available navigation systems are still restricted to applications for rigid structures, such as the skull or the spine. To address this issue, several research groups (e.g. $[1,2,3,4])$ are investigating methods for compensating organ motion during soft tissue interventions. Different approaches for assessing the accuracy of the navigation systems developed in this context have been applied in the published studies. Zhang et al. [2] implanted tumor models containing radio-opaque CT contrast medium into a silicon liver model mounted on a motion simulator and also conducted experiments in swine with agar injections as nodular targets. Kahn et al. [3] evaluated their navigation system in human cadavers, with three different targets: a predefined position within the 
ascending aorta, a calcified plaque in an artery, and the tip of a port catheter. Fichtinger et al. [1] conducted experiments in ventilated swine cadavers and used stainless-steel staples as targets.

In a previous report [4], we introduced a needle-based navigation system for minimally invasive interventions in the liver, in which a real-time deformation model is used to estimate the position of a navigation target point continuously from a set of optically tracked navigation aids (Fig. 1). The overall targeting accuracy of the system was assessed ex-vivo with a respiratory liver motion simulator and was $3.5 \pm 1.1 \mathrm{~mm}$ on average. In this study, we assess the accuracy of our system in-vivo by performing 32 needle punctures in two swine.

\section{Materials und Methods}

\subsection{Navigation System}

Our navigation concept, which has been presented and discussed in detail in previous works (e.g. [4]), is illustrated in Figure 2.1: Prior to the intervention, a set of fiducial needles (navigation aids) is placed in the vicinity of the lesion (i). Next, a planning CT scan is acquired (ii), which is used to plan a trajectory from the skin towards the target (iii). The navigation aids are localized in the CT image and the registration of the CT coordinate system with the tracking coordinate system is performed based on the positions of the fiducial needles (iv). During the intervention, a real-time deformation model is used to continuously estimate the position of the target point from the current positions of the optically tracked navigation aids (v).

\subsection{Accuracy Assessment}

This study was approved by the committee on animal care and research of the Regierungspräsidium Karlsruhe. We injected eight $2 \mathrm{ml}$ agar nodules into the livers of two ventilated swine as tumor models (5\% agar dilution mixed with contrast agent $1: 15 \mathrm{v} / \mathrm{v}$ dilution). Note that a volume of $2 \mathrm{ml}$ corresponds to a diameter of approximately $1.5 \mathrm{~cm}$ for spherical lesions. One medical expert with experience in punctures (E) and one fourth-year medical student (NE)

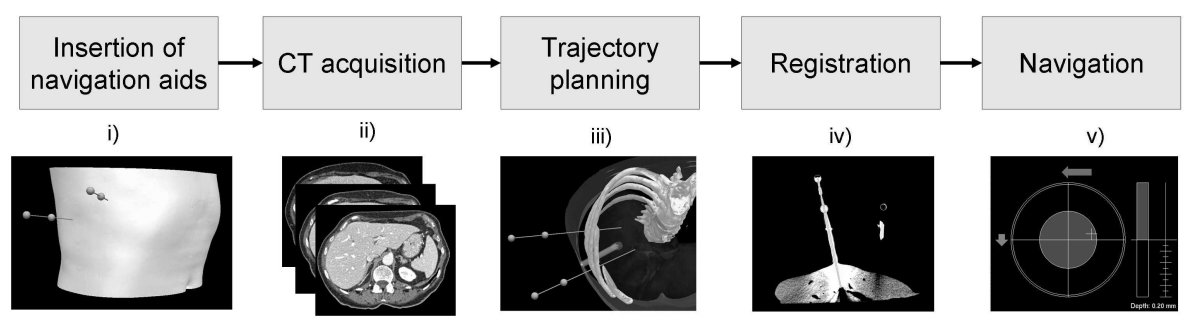

Fig. 1. Soft tissue navigation concept 
with no experience in punctures used the navigation system to target each lesion twice (two passes). For each pass, an ultrasound (US) device was used to insert the navigation aids such that they were arranged along the cranio-caudal axis of the animal and did not pass a lesion. Silicon patches were utilized to attach the needles firmly to the skin. Both subjects then targeted the set of four lesions according to the following procedure: In an initial preparation step, a planning CT scan was acquired (Toshiba Aquilion 16 slice multidetector CT scanner (Toshiba, Tokyo, Japan); $1 \mathrm{~mm}$ slice thickness) showing the entire set of lesions and both navigation aids. Next, the navigation aid models were registered with the planning CT image. For each lesion, the following workflow was then applied:

1. Trajectory planning: The lesion was segmented semi-automatically on the basis of the graph-cut algorithm [5] and the navigation target point was set to the center of gravity of the segmented lesion. Next, an insertion point was chosen on the skin such that the trajectory to the target point did not pass any risk structures.

2. Registration: In order to register the tracking coordinate system with the CT coordinate system, we monitored the movement of the fiducial needles over two breathing cycles. For each recorded sample $k$ (i.e., eight times per second), we computed the landmark-based rigid transformation $\Phi_{k}$ yielding the smallest fiducial registration error (FRE) according to the least square method. A set of corresponding control points distributed along the registered and tracked navigation aids served as landmarks for this purpose. The transformation $\tilde{\Phi}$ yielding the smallest FRE within the two breathing cycles was then chosen as coordinate transformation.

3. Navigation: We used an optically tracked instrument to target the agar nodule with the navigation system. The targeting procedure was conducted at end-expiration in $20 \mathrm{sec}$ intervals, i.e., the intervention was interrupted every

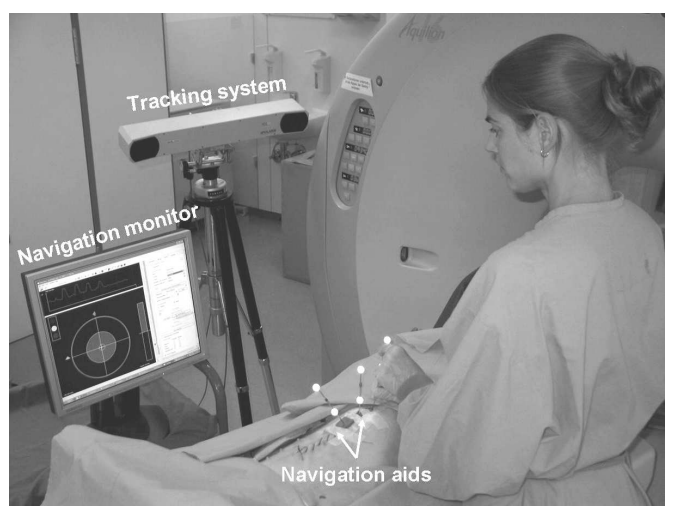

(a)

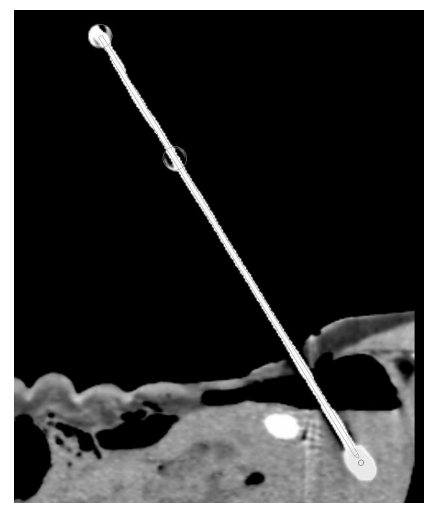

(b)

Fig. 2. Experimental setup (a) and control CT scan (b) showing the registered instrument, the segmented lesion within the liver and the center of gravity of the lesion (circle) 
Table 1. CT targeting error (in $\mathrm{mm}$ ) for the individual trials of the expert (E) and the non-expert (NE).

\begin{tabular}{l|cccc}
\hline Lesion & E, pass & E, pass 2 & NE, pass 1 & NE, pass 2 \\
\hline Swine 1 & & & & \\
L1 & 3.7 & 5.6 & 2.0 & 2.0 \\
L2 & 6.2 & 3.9 & 5.1 & 2.1 \\
L3 & 7.8 & 4.4 & 2.9 & 3.5 \\
L4 & 3.1 & 2.4 & 2.3 & 2.3 \\
\hline Swine 2 & & & & 3.2 \\
L1 & 5.8 & 5.2 & 1.0 & 3.8 \\
L2 & 6.1 & 11.6 & 6.1 & 3.4 \\
L3 & 3.8 & 6.3 & 3.3 & 2.9 \\
L4 & 3.4 & 5.0 & 4.4 & \\
\hline
\end{tabular}

$20 \mathrm{sec}$ to ventilate the swine. A navigation monitor provided the visualization for the targeting process (Fig. 2a).

4. Post-processing: A CT scan was acquired with the same settings as for the planning CT. The lesion in the control CT image was segmented semiautomatically with the graph-cut algorithm [5], and the reference target position was set to the center of gravity of the segmented lesion (Fig. 2b). The instrument model was then registered with the control CT image and the distance between the computed target point and the position of the instrument tip was recorded as the CT targeting error.

\section{Results}

Our navigation system was successfully applied for 32 needle punctures according to the workflow described above. The lesions were successfully hit in $97 \%$ of all trials (31 out of 32) with a mean fiducial registration error (FRE) of $0.9 \pm 0.3 \mathrm{~mm}$ (max: $1.7 \mathrm{~mm}$ ) for computation of the coordinate transformation. The mean final distance between the instrument tip and the center of gravity of the segmented agar nodule was $5.3 \pm 2.2 \mathrm{~mm}(\mathrm{n}=16)$ for the expert, $3.2 \pm 1.3 \mathrm{~mm}(\mathrm{n}=16)$ for the non-expert, and $4.2 \pm 2.1 \mathrm{~mm}(\mathrm{n}=32)$ averaged over both subjects. The results for the individual trials are shown in Table 1. According to the Wilcoxon test, the non-expert obtained significantly better results than the medical expert.

\section{Discussion}

We assessed the targeting precision of a previously reported soft tissue navigation system and obtained a mean targeting error of $4.2 \pm 2.1 \mathrm{~mm}$ for 32 needle punctures. The measured error incorporates the system error, whose main sources are the $\mathrm{CT}$ registration error, the needle tracking error, and the error of the deformation model, and the user error which results from an inaccurate needle 
insertion. It is worth mentioning in this context, that both participants in this study reported a shift of the instrument once they released it to allow for the control CT scan acquisition. Despite the fact that they were allowed to adjust the position until they were satisfied with the targeting error given by the tracking system, optimal positioning was not always possible due to the weight of the instrument and the relatively small layer of tissue between the skin and some of the lesions. One of the lesions shown in Figure 2b, for instance, was located very close to the liver capsule. The non-expert was more successful in inserting the instrument such that it did not move considerably upon release and thus achieved a better user error and therewith CT targeting error.

The main drawback of our navigation concept is the fact that the insertion of two fiducial needles initially increases the invasiveness of an intervention. On the other hand, tracking points inside the target organ for motion compensation potentially leads to high accuracy and can thus eliminate the requirement for repeated needle insertions. In fact, our targeting accuracy is higher than that published in related work. Zhang et al. [2] reported a success rate of $87.5 \%(\mathrm{n}=16)$ in a silicon liver mounted onto a motion simulator and a mean targeting error of $8.3 \pm 3.7 \mathrm{~mm}(\mathrm{n}=32)$ in swine. Other groups obtained mean errors of $8.4 \pm 1.8 \mathrm{~mm}(\mathrm{n}=42)$ in human cadavers [3] and $6.4 \pm 1.8(\mathrm{n}=22)$ in ventilated swine [1].

From a clinical point of view, a robust targeting precision of the order of magnitude of $4 \mathrm{~mm}$ would improve the treatment standard for CT-guided minimally invasive interventions in the liver dramatically. To advance clinical application of our method we are currently conducting a study for comparing the conventional method for CT-guided needle insertions with our approach with respect to accuracy, time, and radiation exposure to the patient.

\section{References}

1. Fichtinger G, Deguet A, Fischer G, et al. Image overlay for CT-guided needle insertions. Comp Aid Surg. 2005;10(4):241-55.

2. Banovac HZF, Lin R, et al. Electromagnetic tracking for abdominal interventions in computer aided surgery. Comp Aid Surg. 2006;11(3):127-36.

3. Khan MF, Dogan S, Maataoui A, et al. Navigation-based needle puncture of a cadaver using a hybrid tracking navigational system. Invest Radiol. 2006;41(10):71320 .

4. Maier-Hein L, Pianka F, Seitel A, et al. Precision targeting of liver lesions with a needle-based soft tissue navigation system. Proc MICCAI. 2007;4792(2):42-9.

5. Boykov Y, Kolmogorov V. An experimental comparison of min-cut/max-flow algorithms for energy minimization in vision. IEEE Trans Pattern Anal Mach Intell. 2004;26(9):1124-137. 